\title{
NMDA-Induced Dendritic Oscillations during a Soma Voltage Clamp of Chick Spinal Neurons
}

\author{
L. E. Moore, ${ }^{1}$ N. Chub, ${ }^{2}$ J. Tabak, ${ }^{2}$ and M. O'Donovan ${ }^{2}$ \\ ${ }^{1}$ Laboratoire de Neurobiologie des Reseaux Sensorimoteurs, UPRESA-7060, Paris, France, and ${ }^{2}$ Laboratory of Neural \\ Control, National Institutes of Health, Bethesda, Maryland 20892
}

An investigation of dendritic membrane properties was performed by whole-cell patch measurements of the biophysical properties of intact chick spinal neurons that are involved in rhythmogenesis. A whole-cell voltage clamp of the somatic membrane was used to block NMDA-induced voltage oscillations from the cell body, thus partially isolating the intrinsic oscillatory properties of dendritic membranes from those of the soma. An experimental approach was developed that takes into account the complexity of the dendritic tree in an environment as normal as possible, without the need for cell isolation or slice preparations. A computational study of the experimentally determined model showed that excitatory amino acid receptors on dendrites can dynamically control the electrotonic length of the dendrites through the activation of negative slope conductances. These experiments demonstrate the presence of NMDA receptors on the dendrites and that they induce intrinsic oscillations when the synaptic input from other cells is significantly reduced.

Key words: dendritic oscillations; chick spinal neurons; NMDA; electrotonic structure; frequency domain; impedance
The determination of dendritic membrane properties has been most successful using direct whole-cell patch studies in both isolated and slice preparations (Major et al., 1993a; Spruston et al., 1993, 1994; Bekkers and Stevens, 1996; Kavalali et al., 1997). Recently, measurements from single neurons before and after isolation have introduced an innovative way to estimate the "removed" dendritic conductances (Destexhe et al., 1998). These techniques (Jackson, 1992; Surmeier et al., 1994; Stuart and Spruston, 1998), in combination with a number of available computational studies, have provided significant theoretical insight into the role of dendritic processing for numerous neuronal behaviors (Traub and Miles, 1991; Mainen et al., 1995; White et al., 1995; Koch and Segev, 1998). As an extension of these methods to neurons in their functioning networks (O'Donovan et al., 1992; Roberts et al., 1995; Marder and Calabrese, 1996; O'Donovan and Chub, 1997), we have developed an approach that takes into account the complexity of the dendritic tree in an environment as normal as possible, without the need for cell isolation or slice preparations. Our approach is fundamentally based on the pioneering work of Rall and his collaborators (Segev et al., 1995) who developed much of the mathematical formalism needed for the analysis of dendritic membrane properties. Our method uses step voltage-clamp currents and small signal frequency domain responses, both of which are elicited by the same final command membrane potential.

Thus, both conventional large-step nonlinear responses as well as small-signal linear perturbations are used to analyze dendritic conductances of intact neurons by explicitly taking into account the currents originating from the unclamped cable processes attached to the soma (Major, 1993; Major et al., 1993b). This

Received March 23, 1999; revised July 8, 1999; accepted July 20, 1999.

This work was supported in part by the Centre National de la Recherche Scientifique (Paris, France).

Correspondence should be addressed to Dr. Lee E. Moore, Laboratoire de Neurobiologie des Reseaux Sensorimoteurs, UPRESA-7060, Centre National de la Recherche Scientifique, 45 rue des Saints-Peres, 75270 Paris Cedex 06, France. Copyright (C) 1999 Society for Neuroscience 0270-6474/99/198271-10\$05.00/0 approach allows an accurate determination of electrotonic parameters, and under certain conditions, the active ionic conductances of the dendrites can be quantitatively described.

An assessment of dendritic properties is especially useful in understanding rhythmogenesis in the embryonic chick spinal cord. Previous experiments (Sernagor et al., 1995; Chub and O'Donovan, 1998) have suggested that excitatory amino acid conductances, which are increased during rhythmic activity, are likely to be primarily dendritic, whereas GABA/glycine conductances are predominantly somatic. These conclusions were based on experiments in which transmitter antagonists injected into the motor nucleus in the vicinity of the cell bodies abolished presumed GABA-A responses but only partially depressed excitatory amino acid responses (Sernagor et al., 1995).

Thus, to quantify the above issues, measured biophysical properties of intact spinal neurons involved in rhythmogenesis of the chick spinal cord were used to construct a quantitative somatic and dendritic model of both the voltage-dependent ionic conductances and those induced by NMDA activation. A whole-cell clamp of the somatic membrane was used to block voltage oscillations from the cell body, thus partially isolating the intrinsic oscillatory properties (Lampl and Yarom, 1997) of dendritic membranes from those of the soma (Seutin et al., 1994). This method was used to demonstrate that NMDA receptors are present on the dendrites and show oscillatory behavior independent of the somatic membrane receptors.

\section{MATERIALS AND METHODS}

Experimental procedures. The experiments were performed on isolated spinal cords of embryonic day 10-11 White Leghorn chicken embryos that had been maintained at $38^{\circ} \mathrm{C}$. As described previously (Chub and O'Donovan, 1998), the spinal cord was prepared in cooled $\left(10-15^{\circ} \mathrm{C}\right)$, oxygenated Tyrode's solution containing (in mM): $139 \mathrm{NaCl}, 12$ glucose, $17 \mathrm{NaHCO}_{3}, 2.9 \mathrm{KCl}, 1 \mathrm{MgCl}$, and $3 \mathrm{CaCl}_{2}$. Measurements were made at $28^{\circ} \mathrm{C}$ with continuous perfusion. When indicated, $20 \mu \mathrm{M}$ NMDA was added to the perfusate. The whole-cell recording was performed with electrode solutions containing (in $\mathrm{mM}$ ): $130 \mathrm{~K}$-gluconate, $10 \mathrm{NaCl}, 10$ HEPES, 1.1 EGTA, $1 \mathrm{MgCl}_{2}, 0.1 \mathrm{CaCl}_{2}, 1 \mathrm{Na}_{2} \mathrm{ATP}$, and $10 \mathrm{QX}-314$ 
(lidocaine, $N$-ethyl bromide quaternary salt). All measurements reported here were performed in the continuous voltage-clamp mode using an Axoclamp 2A (Axon Instruments, Foster City, CA). All other procedures were identical to those described by Chub and O'Donovan (1998).

The whole-cell patch was made with an electrode under positive pressure that was advanced through the ventral white matter of the chick spinal cord until cell bodies were reached. Gigaseals were then made with a slight negative pressure as described previously (Sernagor and O'Donovan, 1991). Although this technique allows recordings from neurons in a completely intact spinal cord, electrode resistances are often increased over their value in the bathing solution before the seal is made. Quantitative measurements require an accurate correction of their effects (Wilson and Park, 1989). Most studies have used bridge-balance techniques, for which there are numerous problems because they are dependent on both the resistive and capacitative properties of the electrode (Burke and Bruggencate, 1971; Jackson, 1992; Wright et al., 1996). In addition, electrode resistances are known to change, which requires reassessment of the bridge balance during the course of an experiment (Armstrong and Gilly, 1992). Thus, unless some evaluation of the active electronic compensation filtering of the signal is performed, it is impossible to determine the accuracy of the measurement. The technique presented here provides a monitor of the electrode properties during each voltage-clamp step.

Our technique consists of a whole-cell large-signal nonlinear stepvoltage clamp on which is superimposed a sum of low-amplitude sine waves that evoke a linear response after all ionic conductance transient responses are complete (Moore and Christensen, 1985, 1987). The analysis is thus a hybrid of linear (Moore et al., 1987, 1993; D'Aguanno et al., 1989 ) and nonlinear methods in both the time and frequency domains (Murphey et al., 1995, 1996). Figure $1 A$ shows the current response to a hyperpolarizing voltage command at the soma electrode, which consists of a step followed by a sum of sines (white noise) superimposed on the step at $0.6 \mathrm{sec}$. The amplitude of the white noise voltage signal was maintained below $3-5 \mathrm{mV}$ root mean square to obtain a linear response. The current response shows an initial transient, attributable to both electrotonic structure and the electrode (see Fig. $1 A$ ). With depolarizing steps, this was followed by second ionic conductance transient (see Fig. $2 A$ ) and finally a steady-state response consisting of both DC and sine wave components (see Fig. $2 A, B$ ). The linear frequency domain responses are ratios of the amplitudes of the voltage and current sine waves and the phase shifts between them for each frequency (see Fig. $1 B$ ). This was performed with a 1024 point Fast Fourier transform on the steadystate data collected from 0.8 to $1.6 \mathrm{sec}$ that was low pass-filtered at 0.4 times the maximum frequency measured (Moore et al., 1988). The transformed data are shown in the figures as magnitude and phase relationships. The analysis is based on data from 18 neurons, all of which show similar results.

Thus, the determination of dendritic properties consists of three parts: (1) large- and small-stimulus voltage- and current-clamp experiments, (2) a parameter estimation that tests different neuronal models, and (3) a computational investigation of the nonlinear predictions of the experimentally determined models. This paper presents an analysis of wholecell voltage-clamp data from real neurons with dendritic trees and demonstrates that dendritic properties can be quantitatively analyzed by this approach.

Computational models. Nonlinear compartmental models were used to quantitatively fit soma voltage-clamp currents (Murphey et al., 1995, 1996) to estimate (1) the electrotonic structure of the dendrites and (2) their voltage-dependent conductances. Because neuronal dendritic structures preclude a space clamp (Armstrong and Gilly, 1992), it is not possible to use the relatively simple analytical solutions of voltage-clamp current responses traditionally used in a Hodgkin-Huxley analysis (Hodgkin and Huxley, 1952). Therefore, the complete nonlinear equations were solved numerically for all real time parameter estimations and simulations of model responses. The models used in the data analysis were required to describe all of the time and frequency domain data from a particular neuron. All models consisted of an electrotonic structure on which uniformly distributed voltage-dependent ionic conductances were expressed. For normal Tyrode's solution in which the sodium conductance was pharmacologically blocked, a potassium conductance $(g k)$ was always used. In addition, most cells required an inward calcium conductance. In the presence of NMDA, the voltage-dependent conductances consisted of an inward NMDA-induced channel (gnmda), the normal potassium conductance $(g k)$, and an additional slower potassium conductance $\left(g k_{\text {slow }}\right)$ that appears only in the presence of NMDA. The slow potassium channel is generally attributed to a calcium-activated channel that is enhanced because of the entry of calcium through the open NMDA channel.

As described above, at each voltage-clamp step a small-signal linear response was evoked. These data were analyzed in the frequency domain with theoretical functions that are the limiting linear response for small perturbations of the complete nonlinear equations (see Figs. $1 B, 2 B$ ). These linear functions do have an analytical form for both the passive and voltage-dependent properties. Previously described parameter estimation methods (Murphey et al., 1995, 1996) were applied simultaneously to both the real-time nonlinear and the linearized data. The constraints imposed by fitting two different types of data provide a more complete test of a particular nonlinear model than an analysis based on one data type (Bhalla and Bower, 1993).

The above considerations illustrate the need to analyze and model as completely as possible the true experimental situation to obtain the correct membrane kinetics that are superimposed on the passive electrotonic structure. The only truly clamped potential is that of the electrode. All other potentials and currents must be described by solutions of the Hodgkin-Huxley-type (Hodgkin and Huxley, 1952), nonlinear differential kinetic equations (Borg-Graham, 1991) appropriate for the experimental condition being analyzed, and with due attention given to the nature of cable equations used to model dendritic processes (Rapp et al., 1994). Two methods were used to model the equivalent dendritic cable: (1) a compartmental model consisting of 3-30 segments in series (D'Aguanno et al., 1989; Murphey et al., 1995, 1996) and (2) a uniform frequency domain analytical model, also with voltage-dependent conductances. This model is equivalent to an infinite number of nonbranched compartments and thus has perfect spatial resolution. In this paper we have shown that a minimal neuronal model, consisting of a soma and one Rall-type equivalent cylinder (Rall and Segev, 1985), quantitatively describes the soma voltage-clamp data presented here. Although complicated branching models (Major and Evans, 1994; Major et al., 1994) could more explicitly represent the morphology, they were not required to quantitatively describe our data. Thus, our ability to accurately fit the sensitive frequency domain data near the resting potential with the analytical model of Equation 1 supports the hypothesis that the Rall equivalent cylinder is a reasonable model for embryonic chick motoneurons. Other neuronal types in the spinal cord might require more complicated models, such as two or more equivalent cylinders with different electrotonic parameters, as discussed by Major et al. (1994).

All analyses in the frequency domain were performed with the uniform analytical model:

$$
Y_{\mathrm{a}}=Y_{\text {soma }}+\frac{g l^{*} A \sqrt{Y_{\text {soma }} / g l}}{L} \tanh \left(L \sqrt{Y_{\text {soma }} / g l}\right),
$$

and

$$
\begin{gathered}
Y_{\text {soma }}(V, f)=j 2 \pi f c_{\text {soma }}+g l+\sum_{\mathrm{p}} g_{\mathrm{p}}\left\{x_{\infty}(V)+\right. \\
\left.\left(V-V_{\mathrm{p}}\right) \tau_{\mathrm{x}}\left(d x_{\infty} / d V\right) /\left(1+j 2 \pi f \tau_{\mathrm{x}}\right)\right\}, \\
Y_{\mathrm{t}}=2 \pi f c_{\mathrm{e}}+Y_{\mathrm{a}} /\left(1+r_{\mathrm{e}} Y_{\mathrm{a}}\right),
\end{gathered}
$$

where $Y_{\mathrm{t}}$ is the total admittance with the electrode, $Y_{\mathrm{a}}$ is the admittance seen at the soma, $Y_{\text {soma }}(V, f)$ represents the passive and active admittances of the soma compartment, $c_{\text {soma }}$ is the soma capacitance, $g l$ is the passive soma conductance, $V_{1}$ is reversal potential for leakage conductance, $L$ is the electrotonic length, $A$ is the ratio of dendritic to soma area, $c_{\mathrm{e}}$ is the electrode capacitance, $r_{\mathrm{e}}$ is the electrode resistance, $j=$ $\checkmark-1, V$ is potential, and $f$ is the frequency in hertz (see below for the remaining definitions).

Parameter estimation. The finite difference method was used to obtain the gradient of the real-time model response to apply nonlinear optimization directly to the dynamic response of the model. The model equations were solved with the backward differentiation integration method (Byrne and Hindmarsh, 1975) or the NDSolve algorithm of Mathematica (Wolfram, 1996). The gradient-type parameter estimation (Dennis et al., 1981) was used to fit the measured admittance spectrum and the solution of ordinary differential equations to the real-time and frequency domain voltage-clamp data. In all cases a simultaneous analysis of the temporal and frequency domain measurements at different membrane potentials was performed. Iterative gradient descent was used until the minima error that was reached changed $<0.1 \%$. Local minimum were avoided by 
verifying that changes in the relative weighting of the frequency and time domain data did not move the fit to a different region in parameter space. Although the final fits were performed by estimating all parameters over a range of membrane potentials, the initial estimates were obtained by the following sequential procedures. (1) By using one or two hyperpolarizing records, seven passive parameters $-c_{\text {soma }}, g_{1}, V_{1}, L, A, c_{\mathrm{e}}$, and $r_{\mathrm{e}}$ were estimated over the measured frequency range. Because $V_{1}$ is not used in the frequency domain (Equation 1), only six parameters are used for this condition. (2) Next, $V_{\mathrm{l}}, r_{\mathrm{e}}$, and $c_{\mathrm{e}}$ were fixed, and the remaining four electrotonic ones $\left(c_{\text {soma }}, g_{1}, L\right.$, and $A$ ) were estimated over one-half of the frequency range of procedure 1. (3) Finally, the voltage-dependent conductance parameters were estimated as in procedure 2 , where $g_{\mathrm{p}}$ is a generic voltage-dependent ionic conductance with a reversal potential of $V_{\mathrm{p}}$ and whose kinetics is governed by the unitless variable $x$, which has a steady-state value of $x_{\infty}$. Thus, at the half-activation $\left(x_{\infty}=1 / 2\right)$ voltage, $v_{x}$, $s_{\mathrm{x}}$ is the slope of $x_{\infty}, t_{\mathrm{x}}$ is the time constant, $\tau_{\mathrm{x}}$, and $r_{\mathrm{x}}$ is the normalized slope of $\tau_{\mathrm{x}}$. In this paper $g_{\mathrm{p}}$ represents potassium ( $\left.g k\right)$-, calcium ( $\left.g c a\right)$-, and NMDA-induced conductances, both inward $\left(g_{\text {nmda }}\right)$ and outward ( $\left.g k_{\text {slow }}\right)$ (Murphey et al., 1995). All fits of active conductances involved a maximum of two conductances, one inward $\mathrm{gCa}$ and one outward $\mathrm{gK}$.

In each of the three steps, all of the time domain data, and the same 50 frequencies out of 400, logarithmically distributed from 0.5 to $250 \mathrm{~Hz}$, were selected for fitting. Thus, low-frequency points were emphasized by this selection, as indicated by the log plots. Procedure 3 was also used in estimating NMDA-induced conductances ( $g_{\text {nmda }}$ and $\left.g k_{\text {slow }}\right)$; however, all other passive and active conductances were kept fixed from previous estimations. This approach prevented mixing the effects of the normal voltage-dependent conductances with those induced by NMDA. Similar to classic voltage-clamp experiments, a combination of voltage ranges, ion substitutions, and the use of pharmacological blocking agents is required to obtain a detailed description of multiple ionic conductances. The advantages of incorporating frequency domain measurements with real-time analysis are numerous: (1) electrotonic structure is more easily taken into account, (2) the presence of voltage-dependent conductances in the dendritic tree can be explicitly evaluated, and (3) combinations of linear and nonlinear responses constrain parameter estimation more than either one alone.

Electrode properties. Before discussing the active and passive parameters, it is necessary to clarify the contribution of the electrode (Armstrong and Gilly, 1992) to the data analysis. This was achieved by simulating the model of Figure 1 with the fitted parameters, but changing just the value of the electrode resistance from its estimated high value of $36 \mathrm{M} \Omega$ (solid lines) to a low one of $0.5 \mathrm{M} \Omega$ (see Fig. $1 A, B$, dashed lines). Under these latter conditions the hyperpolarized current at $-70 \mathrm{mV}$ (see Fig. $1 A$, dashed curve) is indicated as an electrotonic response because the predicted transient is mainly caused by the dendritic cable structure of the model (Spruston and Johnston, 1992). Thus, the near superposition of the two electrode model responses (dashed and solid smooth lines) and data show that the slow component of the essentially passive somatic current is relatively insensitive to the value of the electrode resistance. Therefore, the passive dendritic cable of the neuron contributes more significantly to the hyperpolarizing real-time response than the electrode in series with the soma capacitance.

In contrast to the real time data, the frequency domain plot for the $-70 \mathrm{mV}$ in Figure $1 B$ (dashed lines) shows a dramatic change when the electrode resistance is lowered to 0.5 . Similar high-frequency distortions were also seen with the depolarized frequency domain records (data not shown). It is clear from this simulation that changes in the electrode resistance and capacitance that might occur during an experiment cannot be easily determined from the current transients but are readily accounted for by the frequency domain functions that were measured in the steady-state at each voltage-clamp step.

Although the hyperpolarized time domain data are relatively insensitive to a decrease in the electrode resistance, depolarized voltage-clamp currents are markedly altered (see Fig. $2 A$, dashed curves). The most obvious difference is the steady-state current because the voltage drop across the electrode affects the final membrane potential. The computed initial transients of the low-resistance electrode model at -20 and -10 $\mathrm{mV}$ have a time course similar to that of the measured hyperpolarized current, i.e., significantly faster than the observed slower response during a depolarization. Clearly, the distortions throughout the depolarized transients are caused by an interaction of the turning on kinetics of the active conductances and the passive electrotonic behavior of the neuron plus the electrode (Armstrong and Gilly, 1992; Surmeier et al., 1994; White et al., 1995). Unfortunately, such electrode and similar electrotonic effects do not alter the general shape of the current and could be mistaken for true conductance time courses and therefore misinterpreted.

\section{RESULTS}

\section{Somatic voltage clamp}

The hybrid parameter estimation method was simultaneously applied to hyperpolarized and depolarized potentials, i.e., data sets that were both passive and active. The passive electrotonic parameters were uniquely constrained by the hyperpolarized data (Borst and Haag, 1996), and the voltage-dependent kinetic behavior was expressed through the same electrotonic structure at depolarized potentials. Simultaneous parameter estimation over a range of membrane potentials thus assured a self-consistent model of both the passive electrotonic structure as well as the voltage-dependent conductances. The hyperpolarized voltageclamp currents of Figure $1 A$ were fitted with a passive dendritic compartmental model (smooth curves) having an electrotonic length of 0.5 and a dendritic to soma area ratio of 26. The corresponding frequency domain data of Figure $1 B$ were fitted simultaneously with the identical model in its analytical form. Average passive electrotonic values for seven neurons $( \pm S D)$ for the electrotonic parameters were $c_{\text {soma }}=12.6( \pm 16.3) \mathrm{pF}, L=$ 0.38 ( \pm 0.17$), A=16.4( \pm 8.2), g l=5.6( \pm 14.7) \mathrm{pS}, r_{\mathrm{e}}=29.8$ $( \pm 10.3) \mathrm{M} \Omega, c_{\mathrm{e}}=6.3( \pm 1.8) \mathrm{pF}$.

The depolarized soma voltage-clamp response to $-20 \mathrm{mV}$ in Figure 2 shows a modest outward current; however, the frequency domain functions are dramatically modified in both amplitude and phase compared with the passive hyperpolarized condition (Fig. 1). Together, the time and frequency domain data provide important information about the nature of the voltage-dependent ionic conductances. The magnitude shows a pronounced increase and resonance at a few hertz, and the phase decreases to a negative value below -2 radians (Fig. $2 B$ ), i.e., considerably more negative than $-90^{\circ}(-\pi / 2$ radians $)$. This result can only occur if a negative slope conductance has been activated. Negative slope conductances are generally associated with the activation of an inwardly directed current carried by sodium or calcium ions. The phase function of a conductance showing a positive $I-V$ slope can never be more negative than $-90^{\circ}(-\pi / 2$ radians). Further depolarization to $-10 \mathrm{mV}$ shown in Figure $2 B$ illustrates a shift of the resonance to higher frequencies, with an accompanying sharp phase transition characteristic of highly resonant responses. The step voltage-clamp current at $-10 \mathrm{mV}$ shows an increased outward current suggesting the activation of a putative potassium conductance. These data could be accounted for by an inactivating positive conductance that showed a negative slope for its $I-V$ plot at $-20 \mathrm{mV}$; however, this hypothesis seems unlikely because no indication of a decrease in the outward current during voltageclamp steps was observed. It is plausible to propose an inward calcium conductance to account for the negative conductance because the sodium conductance was blocked by QX314 (Sigma, St. Louis, MO). Thus, the neuronal model fits shown in Figure 2 consist of a putative outward potassium conductance and an inward calcium conductance.

\section{Anomalous impedance increase with NMDA activation}

The addition of $20 \mu \mathrm{M}$ NMDA to the external solution activated rhythmic activity similar to that seen in this preparation either spontaneously or after a brief cathodal pulse to the spinal cord. After some seconds the patterned behavior was replaced by tonic, disorganized activity that appeared to inhibit further synchro- 
WHOLE CELL SOMA VOLTAGE CLAMP

Figure 1. Whole-cell soma voltage clamp. $A$, Current response to a voltage-clamp command. The step voltage command was applied for $0.2-0.6 \mathrm{sec}$, after which was added $1 \mathrm{sec}$ of a small signal deterministic white noise signal consisting of 400 sequential sine waves with randomized phases. The step response from 0 to $0.6 \mathrm{sec}$ is data recorded from the neuron followed by a white noise curve that is a schematic representation of the current response. The smooth curves superimposed on the data represent model responses computed with parameter values fitted simultaneously in the time and frequency domain for a $20 \mathrm{mV}$ hyperpolarizing voltage-clamp pulse from a holding level of $-50 \mathrm{mV}$. The dashed lines are model calculations with only a change in the electrode resistance, $r_{\mathrm{e}}$, from 36 to 0.5 $\mathrm{M} \Omega$. More complicated segmental models for the electrode properties gave a better description of some electrodes; however, the simple lumped model described above provided nearly identical membrane parameter estimates. Therefore, the simpler electrode model was routinely used. B, Fitted magnitude (Megaohms) and phase (radians) functions for each voltage-clamp step are superimposed on measured responses. The dashed lines at $-70 \mathrm{mV}$ represent a model simulation where the electrode resistance was lowered to $0.5 \mathrm{M} \Omega$ as in $A$. All measurements were analyzed from 1.25 to 250 $\mathrm{Hz}$. The parameter values for this neuron are given in Figure 2 (Neuron \#1).
A. Time Domain

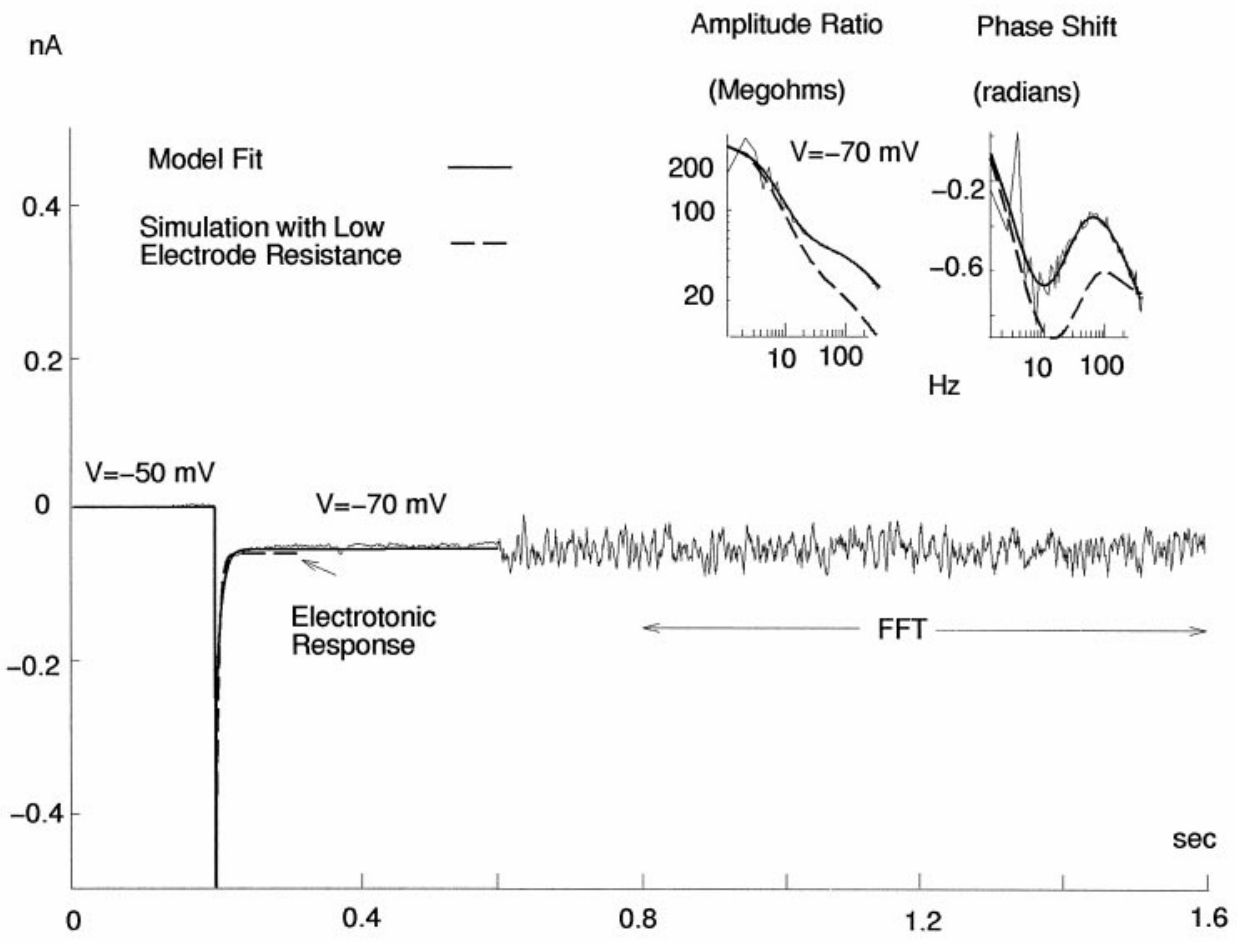

nized responses. Application of TTX at this stage often led (four of five neurons) to the appearance of intrinsic pacemaker activity similar to that observed in other spinal cord preparations (Brodin et al., 1991; Wallén et al., 1992; Scrymgeour-Wedderburn et al., 1997). Voltage-clamp responses from such a preparation are shown in Figure $3 A$ where a maintained NMDA-induced inward current at the holding potential of $-50 \mathrm{mV}$ was observed. A $5 \mathrm{mV}$ step depolarizing pulse elicited a small increase in the negative current; however, a larger $20 \mathrm{mV}$ hyperpolarization slightly reduced it. This type of response was never observed in normal control solutions and was adequately modeled as an NMDAinduced inward conductance in which a magnesium block of the NMDA receptor conferred voltage-dependent properties (Moore and Buchanan, 1993). For this limited polarization range, $g k_{\text {slow }}$ was not needed to model the data, because its activation occurs at more depolarized potentials. The model describes well the reduction of the inward current with hyperpolarization caused by an enhancement of the Mg block of the NMDA receptor.

The corresponding frequency domain records of Figure $3 B$ show that the $-70 \mathrm{mV}$ response has a monotonically decreasing magnitude with frequency that shows no corner frequency representing the membrane time constant, indicating that it must be well below $1.25 \mathrm{~Hz}$, the lowest frequency measured. Such behavior is characteristic of a balance between negative and positive conductances that leads to the anomalous situation of virtually no change of the current in response to a change in the membrane potential, as experimentally observed in Figure $3 A$. Under these conditions the DC impedance, or resistance, approaches infinity, and the magnitude function versus frequency is approximately linear on a log-log Bode plot. Thus, the impedance is mainly capacitative, having a negative phase function at low frequencies, often close to $-\pi / 2$ radians or $-90^{\circ}$; however, its behavior with frequency is very sensitive to the electrotonic structure of the cell. The frequency domain records for the $5 \mathrm{mV}$ depolarization $(V=$ $-45 \mathrm{mV}$ ) are not smooth because of evoked oscillatory instabilities; nevertheless, the phase function appears to be strongly negative, similar to that observed for the negative conductance of Figure 2, seen at a much greater depolarization $(V=-20 \mathrm{mV})$. In normal Tyrode's solution no negative conductance effect was observed over the limited potential range shown in Figure 3. Average values of four neurons $( \pm \mathrm{SD})$ for the peak conductances were $g k=0.00024( \pm 0.00025) \mu \mathrm{S}, g c a=0.0005( \pm 0.0003) \mu \mathrm{S}$, $g_{\text {nmda }}=0.0027( \pm 0.0047) \mu \mathrm{S}$, and $g k_{\text {slow }}=0.0016( \pm 0.0019) \mu \mathrm{S}$.

\section{Model predictions of experimental results}

In the previous section parameter estimation methods were used to obtain the best fits from the real time and frequency domain data. By contrast, the following analysis consists of model predictions and simulations at more depolarized membrane potentials based on the voltage-clamp analysis presented above. These computational studies consist of both current and somatic voltageclamp predictions and provide an interpretation of observed experimental results, where NMDA-induced oscillatory effects are prominent. 


\section{A. Time Domain}

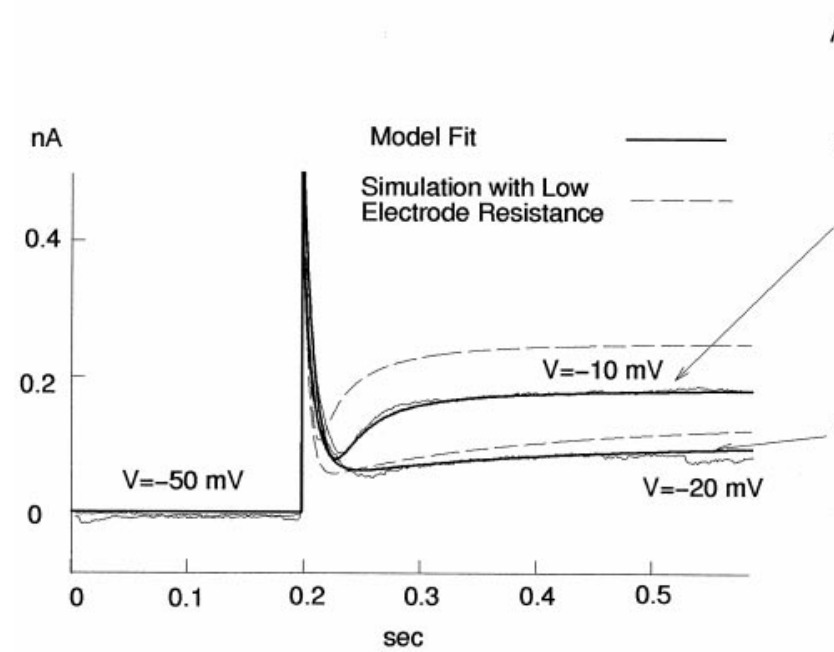

\section{B. Frequency Domain}

Amplitude Ratio

(Megohms)
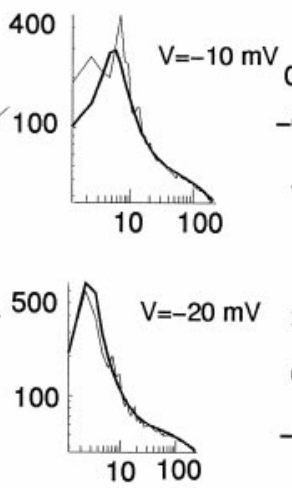

Phase Shift (radians)

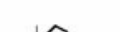

Figure 2. Depolarizing soma voltageclamp currents. The entire data sets, both time $(A)$ and frequency $(B)$ domains, were simultaneously fitted with a neuronal model consisting of an isopotential soma to which were attached the electrode resistance, $r_{\mathrm{e}}$, with its capacitance, $c_{\mathrm{e}}$, plus a dendritic cable as follows: (1) in the frequency domain, an analytical description with a finite length, and (2) in the time domain, a three-compartment model required for the solution of the nonlinear differential equations that describe the voltagedependent conductances (Murphey et al., 1995). The superimposed voltageclamp currents and model fits were elicited from a holding potential of $-50 \mathrm{mV}$ followed by steps to the indicated potentials. The parameter values for the model fits were $c_{\text {soma }}=4.9 \mathrm{pF}, L=0.45$, $A=25.79, g_{1}=0.00012 \mu \mathrm{S}, r_{\mathrm{e}}=36.5$ $\mathrm{M} \Omega, c_{\mathrm{e}}=8.3 \mathrm{pF}, V_{1}=-47.9 \mathrm{mV}, g k=$ $0.00014 \mu \mathrm{S}, v_{\mathrm{n}}=-20.95 \mathrm{mV}, s_{\mathrm{n}}=$ $0.0688 \mathrm{mV}^{-1}, t_{\mathrm{n}}=0.259 \mathrm{sec}, r_{\mathrm{n}}=-0.2$ $\mathrm{mV}^{-1}, g c a=0.0003 \mu \mathrm{S}, r_{\mathrm{s}}=0, v_{\mathrm{s}}=-18$ $\mathrm{mV}, s_{\mathrm{s}}=0.07 \mathrm{mV}^{-1}, t_{\mathrm{s}}=10 \mu \mathrm{sec}$, $V c a=0, V k=-95 \mathrm{mV}$ (Neuron \#1).

\section{Dynamic electrotonic length: dendritic potential profile}

The data analysis showed that the NMDA-induced conductances, as well as presumed intrinsic calcium conductances, lead to extremely large low-frequency impedances. To take into account the role of steady-state active conductances on electrotonic behavior, it is useful to define an effective electrotonic length as follows:

$$
L_{\text {eff }}=L^{*} V\left(\operatorname{Mag}\left[Y_{\text {soma }}(V, f)\right] / g l\right),
$$

where $\operatorname{Mag}\left[Y_{\text {soma }}(V, f)\right]$ is the magnitude of $Y_{\text {soma }}(V, \mathrm{f})$ (see Eq. 2). Thus, $L_{\text {eff }}$ takes into account the capacitance as well as the voltage-dependent conductances and reverts to $L$ at $f=0$ for a passive neuron. The consequence of a nearly infinite intrinsic resistance $(f=0)$ is that $Y_{\text {soma }}(V, 0)$ and therefore $L_{\text {eff }}$ approach zero. Thus, at low frequencies the neuron becomes isopotential, and all maintained peripheral synaptic activity is virtually unattenuated at the soma. At high frequencies $L_{\text {eff }}$ increases, and significant attenuation will occur. To test these conclusions, model simulations are presented to clarify the mechanisms that lead to these unusual effects.

Figure $3 C$ shows that the superimposed voltage-clamp currents for models with 3 and 30 dendritic compartments are remarkably similar, indicating that errors caused by spatial resolution of the three-compartmental model of Figure $3 A$ (smooth curves) are minimal for these parameter values. The hyperpolarizing potential profile of the last dendritic compartment (Fig. 3D) compared with the soma shows a marked delay in the development of a steady-state offset potential. This delay and offset are related to the hyperpolarizing somatic current time course shown for both the model and data, as would be expected of a simple passive cable (Rall, 1960).

By contrast, the depolarization clamp to $-20 \mathrm{mV}$ shows the remarkable result that the steady-state potentials of the soma and last dendritic compartments are nearly the same, the latter having a damped overshoot (Fig. 3D). The clamp control of the soma potential is similar to the hyperpolarizing response, showing a slight delay attributable to the charging of the soma capacitance through the electrode. The computational result of virtually no steady-state current or potential drop between the soma and last dendritic compartment for a somatic step depolarization to -20 $\mathrm{mV}$ is a manifestation of a steady-state negative conductance that increases the effective membrane impedance and thereby reduces the effective electrotonic length $\left(L_{\text {eff }}\right)$ of the dendrite (Müller and Lux, 1993; Moore et al., 1994, 1995). The dramatic nature of this computation is partly attributable to the lack of a large potassium conductance in the model parameters, which would counteract the negative conductance. Nevertheless, these calculations show that this phenomenon provides a means to dynamically control the space constant and significantly alter the effect of synaptic inputs from peripheral dendritic regions (Buchanan et al., 1992; Stuart and Sakmann, 1995).

These results demonstrate that the analysis of point voltageclamp experiments requires a consideration of at least three conditions: (1) the electrotonic structure of the passive dendrite and electrode properties, (2) an increase of the effective electrotonic length $\left(L_{\text {eff }}\right)$ caused by the activation of positive conductances, and (3) a decrease in the effective electrotonic length $\left(L_{\text {eff }}\right)$ because of an enhancement of the low-frequency impedance caused by the activation of negative voltage-dependent conductances. In addition, these changes in the electrotonic length are truly dynamic and must be quantitatively accounted for if somatic voltage-clamp currents are described.

\section{Dendritic membrane potential oscillations in somatic voltage clamp}

The instabilities inferred from Figure $3 B$ for the $5 \mathrm{mV}$ cathodal step are fully developed by the larger depolarizations illustrated 
NMDA Induced Impedance Increase

A. Compartmental Model Fits

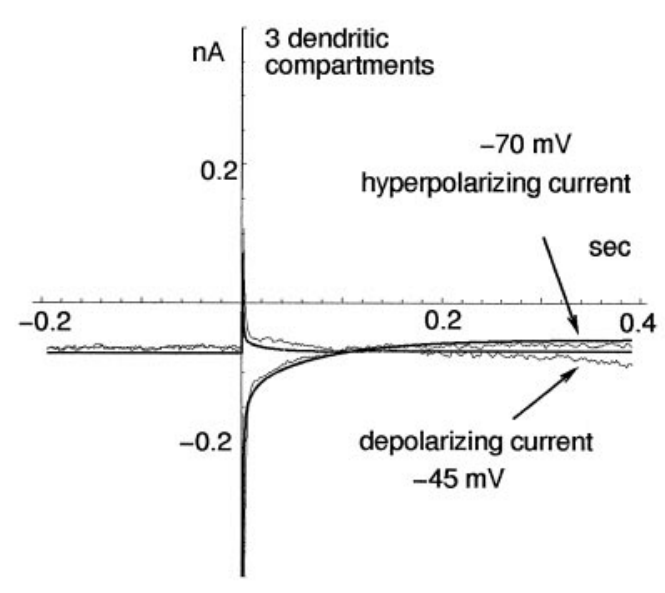

C. Compartmental Model Simulations

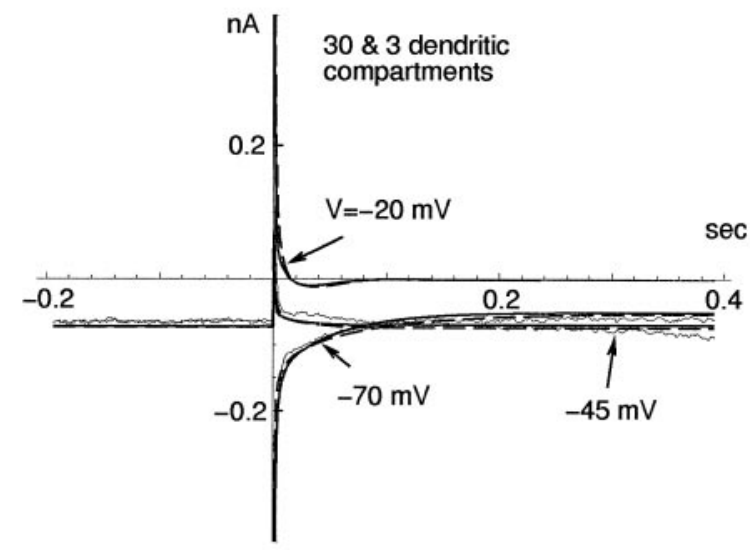

B. Analytical Model Fits
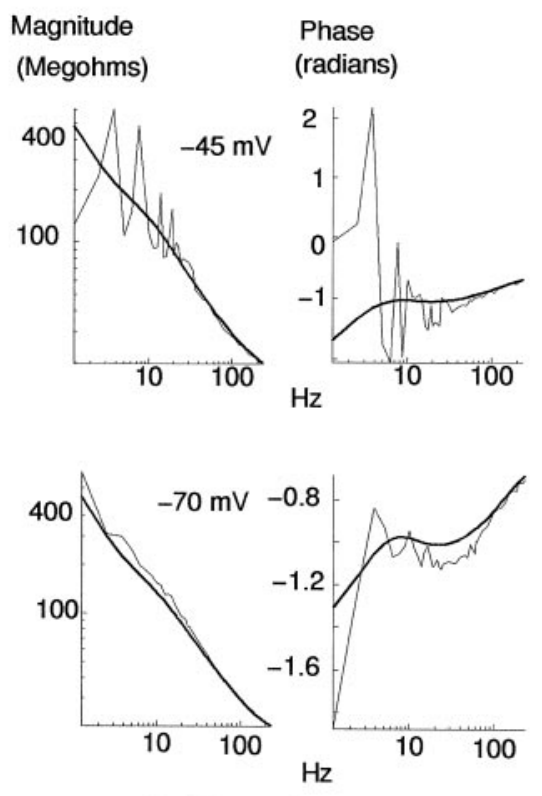

D. Voltage Clamp Potential Profiles

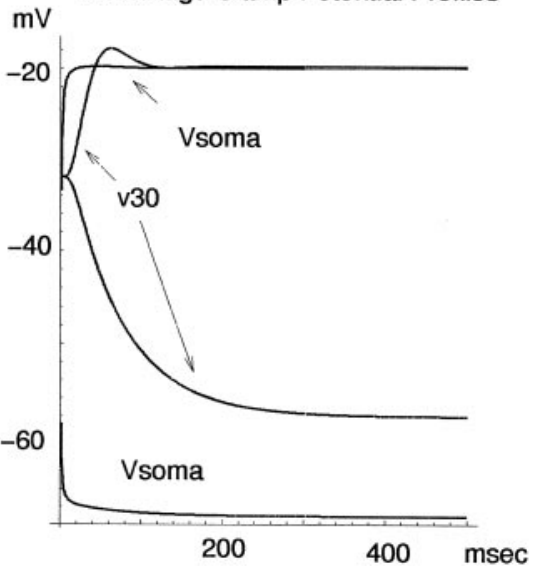

Figure 3. NMDA-induced impedance increase. The soma voltage-clamp responses of $A$ show that the hyperpolarizing current at $-70 \mathrm{mV}$ is less negative than the depolarizing response at $-45 \mathrm{mV}$ elicited from a holding level of $-50 \mathrm{mV}$. This reversal of the usual current response was modeled as a voltage-dependent NMDA-induced current that turns off because of a magnesium block at hyperpolarized potentials. $B$, This conductance has a negative current-voltage relationship that manifests itself in a small-signal frequency domain measurement as a large negative phase at low frequencies. The model responses are superimposed on the data for both time and frequency domain records. The parameter values were $c_{\text {soma }}=49$ $\mathrm{pF}, V_{1}=-39 \mathrm{mV}, g_{1}=0.0002 \mu \mathrm{S}, r_{\mathrm{e}}=18 \mathrm{M} \Omega, c_{\mathrm{e}}=7 \mathrm{pF}, A=4.1, L=0.67, g k=0.0028 \mu \mathrm{S}, v_{\mathrm{n}}=-2 \mathrm{mV}, s_{\mathrm{n}}=0.02 \mathrm{mV}^{-1}, t_{\mathrm{n}}=0.014 \mathrm{sec}, r_{\mathrm{n}}=-0.02$, $g_{\text {nmda }}=0.01 \mu \mathrm{S}, v_{\mathrm{m}}=-5 \mathrm{mV}, s_{\mathrm{m}}=0.02 \mathrm{mV}^{-1}, t_{\mathrm{m}}=0.0001 \mathrm{sec}, r_{\mathrm{m}}=0, V_{n m d a}=0, V k=-95, g c a=0, g k_{\text {slow }}=0.0$. The electrode was modeled with a series resistance, $r_{\mathrm{e}}$, and a capacitance to ground, $c_{\mathrm{e}}$. $C$, The superposition of the 3 and 30 compartmental models are virtually indistinguishable for the three voltage-clamp pulses. The dashed lines represent the 30-compartmental model. The adequacy of the spatial resolution of the compartmental model was evaluated by computing the time domain responses in models having 30 dendritic segments. The electrotonic parameters and distribution of active voltage-dependent conductances of all models were identical. The relationship between the compartmental and analytical models is given by the following formulation in which $x$ is a generalized kinetic variable (Moore and Buchanan, 1993; Murphey et al., 1995): $I_{\mathrm{i}}=I_{\mathrm{p}}+I_{\text {core }}+I_{1}, I_{1}=g_{1}\left(V_{\mathrm{i}}-\right.$ $\left.V_{\mathrm{i}}\right), I_{\mathrm{p}}=\Sigma_{\mathrm{p}} g_{\mathrm{p}} \times\left(V_{\mathrm{i}}-V_{\mathrm{p})}, I_{\text {core }}=g_{\text {core }}\left(V_{\mathrm{i}}-V_{\mathrm{i}+1}\right), I_{\text {core }}=g_{\text {core }}\left(V_{\mathrm{i}}-V_{\mathrm{i}+1}\right)\right.$ and $\mathrm{d} V_{\mathrm{i}} / \mathrm{dt}=-\mathrm{N} \mathrm{I}_{\mathrm{i}} / A c_{\text {soma }}$, where $c_{\text {soma }}$ is the capacitance of the soma, $I_{\mathrm{i}}$ is the current in the ith compartment, $I_{\text {core }}$ is the current between compartments, $V_{\mathrm{i}}$ is the membrane potential in the ith compartment, $g_{1}$ and $I_{1}$ represent a nonspecific leakage conductance and current having $V_{1}$ as a reversal potential, $L$ is the electrotonic length, $A$ is the ratio of dendritic to soma area, $\lambda=$ $N / L$ is in units of the number of compartments, $N$, and $\lambda / \mathrm{g}_{\text {core }}=L /\left(A^{*} \mathrm{gl}\right)$. $D$, The computed potential profiles for the soma and most distal 30 th compartment show that the membrane potential is relatively uniform when the soma was depolarized to $-20 \mathrm{mV}$, which suggests that a smaller number of compartments is adequate for this condition. The similarity of the model behavior in $A$ and $C$ for 3 versus 30 compartments supports this hypothesis. This effect is most striking because $-20 \mathrm{mV}$ is just the potential at which nearly zero net current occurs, giving a ratio of the voltage to current of virtually infinity, i.e., a limiting electrotonic length of zero and a perfect steady-state space clamp. At hyperpolarized levels the conductances are essentially turned off; thus even if there is a potential profile it does not lead to different impedances for the different compartments as would be the case with activation of voltage-dependent positive conductances (Neuron \#2). 


\section{DENDRITIC OSCILLATIONS DURING SOMA VOLTAGE CLAMP}

\section{A. Soma Voltage Clamp}

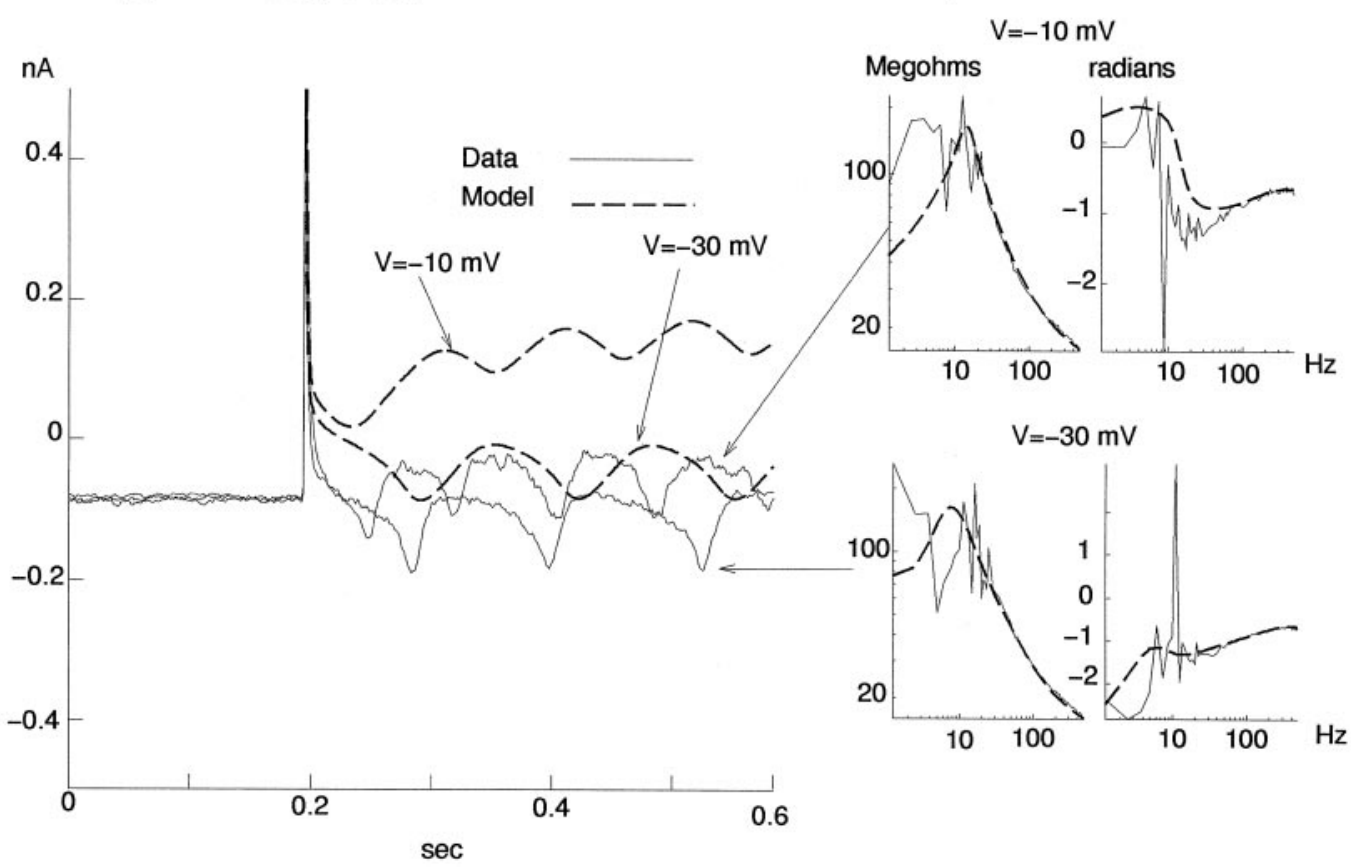

Figure 4. Dendritic oscillations during soma voltage clamp. $A$, The oscillatory currents for somatic depolarizations to -30 and $-10 \mathrm{mV}$ of $A$ are superimposed with model simulations using a dendrite with three compartments in which the $g_{\text {nmda }}$ conductance was increased along with the addition of a slow potassium conductance $\left(g k_{\text {slow }}\right)$ to represent a calcium-activated potassium conductance induced by the influx of calcium during NMDA activation. The dashed model curves are simulations of both the frequency and time domain data using the parameter values of Figure 3 with the following replacements and additions: $g_{\text {nmda }}=0.03 \mu \mathrm{S}, s_{\mathrm{m}}=0.033 \mathrm{mV}^{-1}, g k_{\text {slow }}=0.015 \mu \mathrm{S}, v_{\mathrm{q}}=-5.25, s_{\mathrm{q}}=0.065 \mathrm{mV}^{-1}, t_{\mathrm{q}}=0.25 \mathrm{sec}$, and $r_{\mathrm{q}}=0(\mathrm{Neuron} \# 2)$.

in Figure $4 A$ (solid lines) where large oscillatory currents from the unclamped dendritic regions provide a major contribution to the somatic voltage-clamp currents. The difference in periods of the oscillations observed between those at -30 and $-10 \mathrm{mV}$ is likely to be a consequence of the voltage-dependent $\mathrm{Mg}$ block of the NMDA receptor. Furthermore, the observation of current oscillations during a somatic voltage clamp suggests that the dendritic membrane possesses NMDA receptors. To support this conclusion, model simulations are shown below to confirm that the soma is adequately voltage-clamped.

These oscillatory responses are partially synchronized by the voltage-clamp step and add a nonlinear component to the response during the white noise measurement. At these depolarized levels, parameter estimation could not be performed for the frequency domain data, because the nonlinear uncontrolled responses prevented the achievement of a true linear steady state. As such, this invalidates the assumptions implicit in linear analysis; however, a coherence subtraction procedure was applied to these data to obtain a partial estimation of the frequency domain results. This consists of subtracting current responses of two voltage-clamp steps to the same potential, but with inverted white noise command stimuli. Thus, the identical transient mean currents are cancelled, but the small-signal white noise responses are added, i.e., the coherent responses are eliminated and the smallsignal data are averaged for two voltage-clamp steps. Despite the removal of the mean oscillating response, it is likely that potential dependent fluctuations are present in the small-signal response and would not be accounted for in a linear analysis. Nevertheless, this approach provides empirical estimates of the magnitude and phase functions illustrated in Figure $4 B$. Although there are clear distortions, the results are similar to that seen for smaller depolarizations, for example, a clear negative phase at $-30 \mathrm{mV}$ exceeding $-\pi / 2$ radians. The analytical frequency domain model responses (Fig. $4 B$, dashed lines) at somatic voltage-clamp steps to -30 and $-10 \mathrm{mV}$ show rather good agreement with the data. In addition, the computational model gives resonant frequencies (Moore and Buchanan, 1993) related to the period observed in the real time nonlinear current response.

Because the somatic voltage currents were oscillatory, parameter estimation was not easily performed in the time domain. Therefore, model simulations of the depolarized voltage-clamp responses of Figure $4 A$ (dashed lines) were performed using parameters estimated from the lower step depolarizations of Figure 3 . In addition, a slow potassium conductance, $g k_{\text {slow }}$, was added because of the increased levels of depolarization. Oscillatory responses in the dendrites during a somatic voltage clamp were found for $g_{\text {nmda }}=0.03 \mu \mathrm{S}$ and $g k_{\text {slow }}=0.015 \mu \mathrm{S}$.

\section{Membrane potential oscillations in current clamp}

The oscillatory behavior is markedly different when the experimental condition is changed from measuring somatic current at a fixed soma potential to the current-clamp condition measuring somatic potential at a constant current. This is not too surprising because the voltage clamp clearly restricts the normal intrinsic oscillation by holding the soma potential constant. The somatic current oscillations observed during a voltage clamp are relatively sinusoidal (Fig. 4A); however, the soma membrane potential oscillations observed under current clamp illustrated in Figure $5 A$ 


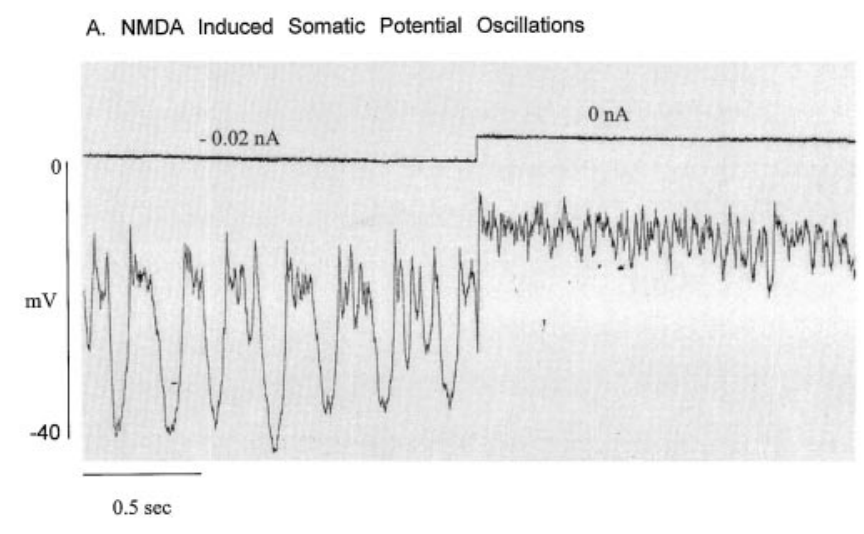

B. Constant Current Simulations

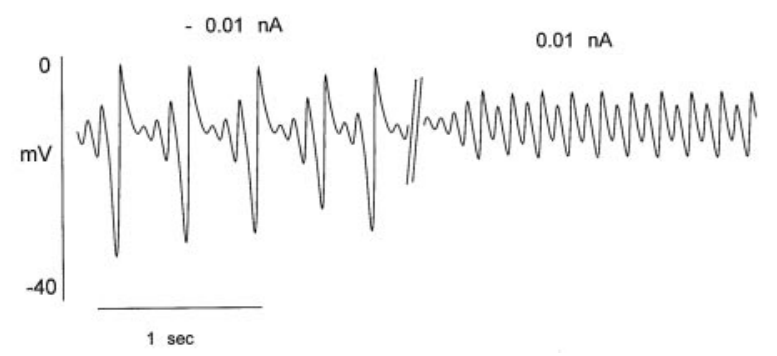

Figure 5. Somatic voltage oscillations during a constant current. $A$, Voltage oscillations in motoneurons at two different current levels. The current levels were 0 and $-0.02 \mathrm{nA}$. $B$, Model simulations with symmetrical polarizing currents $I=-0.01$ and $+0.01 \mathrm{nA}$ show a change from large plateau oscillations to lower amplitude high-frequency behavior, as observed experimentally in $A$. To model the more complex oscillatory behavior seen in current-clamp measurements, $g_{\text {nmda }}$ was increased to 0.06 $\mu \mathrm{S}$. All other parameter values were identical to those of Figure 4. All simulations of Figures 5 and 6 had 30 dendritic compartments.

are more complex. The shape of the soma potential oscillations was markedly dependent on the potential level similar to that observed in the lamprey spinal cord (Wallén et al., 1992). During a hyperpolarization current of $-0.02 \mathrm{nA}$, the rhythmic activity was irregular, having an oscillatory plateau phase whose amplitude was $\sim 20 \mathrm{mV}$ and a duration of $0.2-0.3 \mathrm{sec}$. The plateau oscillations were of low amplitude and relatively high frequency. When the holding current was removed, the membrane potential remained at its plateau level of approximately $-5 \mathrm{mV}$ and showed only the high-frequency oscillations. All oscillations were abolished by hyperpolarizations greater than $-50 \mathrm{mV}$ in both current and voltage clamp.

\section{DISCUSSION}

The observation of oscillatory currents during NMDA activation of whole-cell voltage-clamped neurons shows that the dendritic membrane is well supplied with NMDA receptors. The analysis of time and frequency domain data allowed the development of an electrotonic model of chick motoneurons that can account for the current oscillations during a voltage clamp. The voltage clamp controlled the intrinsic oscillations near the resting potential. Under these conditions an initial estimate of the NMDA-induced conductances was performed. At moderate voltage-clamp depolarizations, oscillatory behavior was observed in the current response that could be simulated by the estimated neuronal model if the maximal NMDA-induced conductance was increased approximately threefold.

A computational investigation of the model behavior can explain the striking difference between the nature of the oscillatory behavior in current versus voltage clamp. For the model to simulate the constant-current plateau oscillations seen experimentally, the NMDA conductance was increased another twofold, with all other parameters the same as in the voltage-clamp simulations of Figure 4. Under these conditions, a hyperpolarization of the model with $-0.01 \mathrm{nA}$ leads to a high-amplitude oscillation with a distinct plateau phase (Fig. $5 B$ ). A depolarizing current of $0.01 \mathrm{nA}$ abolishes the plateau of the model and sustains a highfrequency oscillation, analogous to that observed experimentally (Fig. $5 A$ ). The increase of $g_{\text {nmda }}$ used in the successive modeling steps was attributable to an inability to predict the maximal conductance from data taken near the holding potential, where the responses were stable and parameter estimation was more effective. This illustrates the advantage of simulation studies to obtain parameter values that are needed for a particular behavior and could then be used as constraints for other experimental conditions.

In contrast to the current-clamp plateau oscillations (Fig. 5), the simulated voltage-clamp currents (Fig. $6 A, I_{\mathrm{m}}$ ) are relatively sinusoidal at a soma depolarization to $-30 \mathrm{mV}$, similar to that observed experimentally (Fig. 4A). Thus, this model with two uniformly distributed potassium conductances, fast and slow, in combination with a significant NMDA conductance, can reasonably well simulate the experimental findings of complex plateau oscillations whose low-frequency component is reduced by a voltage clamp of the soma. Furthermore, these computations indicate that the oscillatory voltage-clamped somatic currents are a consequence of dendritic membrane potential (Fig. 6A, $V_{\mathrm{m}}$ ) oscillations (Seutin et al., 1994; Li et al., 1996). This conclusion is dependent on an accurate determination of the electrotonic structure and electrode effects that allow a rigorous assessment of the voltage-clamp control of the soma. Previous simulations performed with only one potassium conductance under constant current conditions (Moore and Buchanan, 1993; Murphey et al., 1995) showed plateau oscillations without superimposed highfrequency oscillations. Thus, the present computations suggest that complex oscillatory patterns require at least three voltagedependent conductances.

During a constant current, the potentials of the soma and peripheral dendritic compartments show little difference between models with 3 and 30 dendritic segments, indicating that spatial resolution is adequate. Under the conditions of strong intrinsic oscillations, whatever their shape, each compartment is well synchronized such that there is essentially no difference between the different locations on the cable. This would not necessarily occur if there were a nonuniform distribution of channel receptors. The 1-2 $\mathrm{mV}$ oscillation seen in the soma (Fig. $6 A$ ) is a consequence of the imperfect soma clamp attributable to an electrode resistance. Figure $6 B$ shows constant current simulation where the soma potential is unclamped such that at zero current all compartments including the soma oscillate in synchrony. During a steady-state hyperpolarization with $-0.05 \mathrm{nA}$ of current, the plateau amplitude is enhanced and the potential profiles from different compartments are not identical; however, the oscillations continue to be well synchronized (Fig. 6B).

In conclusion, the finding that complex plateau membrane potential oscillations observed in current clamp could not be completely abolished by a soma voltage clamp is a consequence of 
Voltage Clamp versus Constant Current Oscillations

\section{A. VOLTAGE CLAMP}

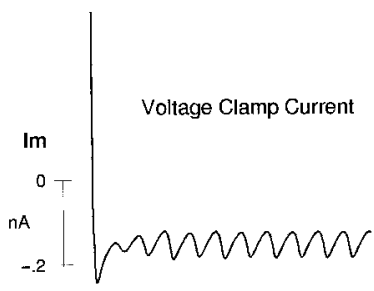

Sinusoidal Current Oscillations from clamped soma membrane

Soma Voltage Clamp

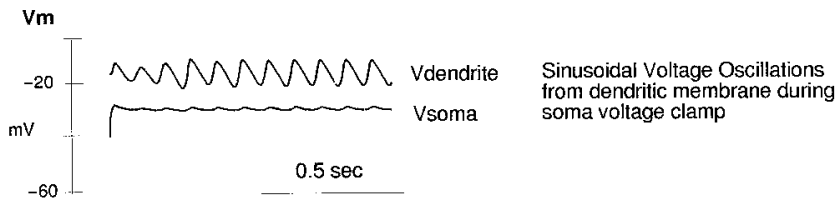

B. CURRENT CLAMP

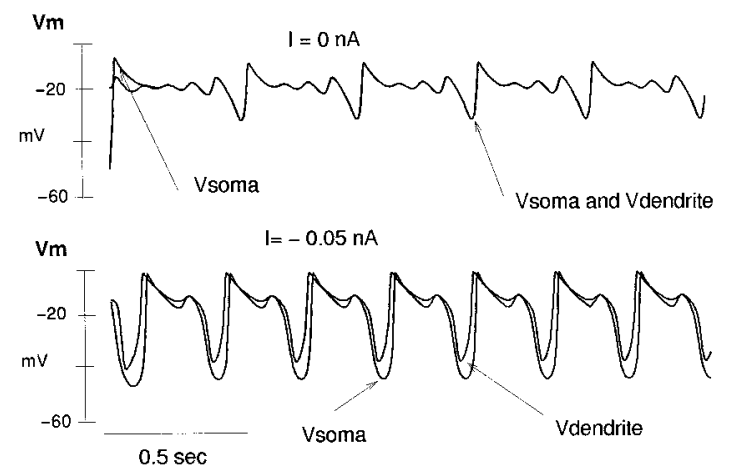

Figure 6. Voltage-clamp versus constant-current oscillations. $A$, The computed somatic voltage-clamp currents $(\mathrm{Im})$ at $-30 \mathrm{mV}$ from a holding level of $-50 \mathrm{mV}$, although increased, remain reasonably sinusoidal, similar to those of Figure $4 A$ at a lower $g_{\text {nmda }}$. The parameter values are identical to those of Figure 5. $B$, The subsequent current clamp (Im $=0$ $\mathrm{nA})$, computed with the initial conditions at $-50 \mathrm{mV}$ of the voltage clamp, shows complex soma and dendritic potential oscillations $(\mathrm{Vm})$. In the bottom panel, current-clamp simulations with a hyperpolarizing current of $-0.05 \mathrm{nA}$ show an enhanced amplitude of the oscillatory plateau phase.

the impossibility of space clamping the dendritic tree. The experimental and computational studies demonstrate the presence of intrinsic dendritic membrane potential oscillations that are significantly different under current- versus voltage-clamp conditions. This behavior provides a means to quantitatively distinguish between soma and dendritic NMDA receptors and can provide an additional constraint in the selection of neuronal models and their parameter values. Thus, parameter estimation, using a combination of current- and voltage-clamp measurements in both the time and frequency domains, allows a nearly unique estimation of dendritic and soma conductances.

Finally, the experimental finding of oscillatory responses during a voltage clamp of the soma definitively demonstrates that NMDA receptors must be present on the dendrites and provides support for an earlier hypothesis that the presence of excitatory amino acid receptors on dendrites is important for rhythmic activity (Chub and O'Donovan, 1998). One important way in which they may act is to reduce the electrotonic length of the dendrites during increased activity by dynamically altering the relative impedance of the cable structures based on the activation of negative conductances, which in turn modifies synaptic efficacy. In addition, when the activation of NMDA receptors is sufficiently large, intrinsic membrane oscillations are established that are likely to stabilize rhythmic patterns.

\section{REFERENCES}

Armstrong CM, Gilly WF (1992) Access resistance and space clamp problems associated with whole-cell patch clamping. In: Methods in enzymology (Rudy B, Iversen LE, eds), pp 100-122. San Diego: Academic.

Bekkers JM, Stevens CF (1996) Cable properties of cultured hippocampal neurons determined from sucrose-evoked miniature EPSCs. J Neurophysiol 75:1250-1255.

Bhalla US, Bower JM (1993) Exploring parameter space in detailed single neurons models: simulations of the mitral and granule cells of the olfactory bulb. J Neurophysiol 69:1948-1965.

Borg-Graham L (1991) Modeling the non-linear conductances of excitable membranes. In: Cellular neurobiology: a practical approach (Chad J, Wheal H, eds), pp 247-275. New York: Oxford UP.

Borst A, Haag J (1996) The intrinsic electrophysiological characteristics of fly lobula plate tangential cells: I. passive membrane properties. J Comp Neurosci 3:313-336.

Brodin L, Traven G, Lansner A, Wallén P, Ekebert O, Grillner S (1991) Computer simulations of $N$-methyl-D-aspartate receptor-induced membrane properties in a neuron model. J Neurophysiol 66:473-484.

Buchanan J, Moore LE, Wallén P, Hill R, Grillner S (1992) Synaptic transfer function of Mueller axon to spinal neuron in lamprey. Biol Cybern 67:123-131.

Burke RE, Bruggencate GT (1971) Electrotonic characteristics of alpha motoneurones of varying size. J Physiol (Lond) 212:1-20.

Byrne GD, Hindmarsh AC (1975) A polyalgorithm for the numerical solution of ordinary differential equations. ACM Trans Math Software 1:71-96.

Chub N, O’Donovan MJ (1998) Blockade and recovery of spontaneous rhythmic activity after application of neurotransmitter antagonists to spinal networks of the chick embryo. J Neurosci 18:294-306.

D’Aguanno A, Bardkjian BL, Carllen PL (1989) A system model for investigating passive electrical properties of neurons. Biophys $\mathrm{J}$ 55:1169-1182.

Dennis JE, Gay DM, Welsch RE (1981) An adaptive nonlinear leastsquares algorithm. ACM Trans Math Software 7:348-368.

Destexhe A, Neubig M, Ulrich D, Huguenard (1998) Dendritic lowthreshold calcium currents in thalamic relay cells. J Neurosci 18:3574-3588.

Hodgkin AL, Huxley A (1952) A quantitative description of membrane current and its application to conduction and excitation in nerve. J Physiol (Lond) 117:500-544.

Jackson MB (1992) Cable analysis with the whole-cell patch clamp. Theory and experiment. Biophys J 61:756-766.

Kavalali ET, Zhuo M, Bito H, Tsien RW (1997) Dendritic Ca 2+ channels characterized by recordings from isolated hippocampal dendritic segments. Neuron 18:651-663.

Koch C, Segev I (1998) Methods in neuronal modeling. Cambridge, MA: MIT.

Lampl I, Yarom Y (1997) Subthreshold oscillations and resonant behavior: two manifestations of the same mechanism. Neuroscience 78:325-341.

Li YX, Bertram R, Rinzel J (1996) Modeling $N$-methyl-D-aspartateinduced bursting in dopamine neurons. Neuroscience 71:397-410.

Mainen ZF, Joerges J, Hugenard JR, Sejnowski TJ (1995) A model of spike initiation in neocortical pyramidal neurons. Neuron 15:1427-1439.

Major G (1993) Solutions for transients in arbitrarily branching cables: III. Voltage clamp problems. Biophys J 65:469-491.

Major G, Evans JD (1994) Solutions for transients in arbitrarily branchings cables: nonuniform electrical parameters. Biophys J 66:615-634.

Major G, Evans JD, Jack JB (1993a) Solutions for transients in arbitrarily branching cables: I. Voltage recording with a somatic shunt. Biophys J 65:423-449.

Major G, Evans JD, Jack JB (1993b) Solutions for transients in arbitrary branching cables: II. Voltage clamp theory. Biophys J 65:450-468.

Major G, Larkman AU, Jonas P, Sakmann B, Jack JJB (1994) Detailed 
passive cable models of whole-cell recorded CA3 pyramidal neurons in rat hippocampal slices. J Neurosci 14:4613-4638.

Marder E, Calabrese RL (1996) Principles of rhythmic motor pattern generation. Physiol Rev 76:687-717.

Moore LE, Buchanan J (1993) The effects of neurotransmitters on the integrative properties of spinal neurons of the lamprey. J Exp Biol 175:89-113.

Moore LE, Christensen BN (1985) White noise analysis of cable properties of neuroblastoma cells and lamprey central neurons. J Neurophysiol 53:636-651.

Moore LE, Hill RH, Grillner S (1987) Voltage clamp analysis of lamprey neurons: role of $N$-methyl-D-aspartate receptors in fictive locomotion. Brain Res 419:397-402.

Moore LE, Yoshii K, Christensen BN (1988) Transfer impedances between different regions of branched excitable cells. J Neurophysiol 59:689-705.

Moore LE, Hill RH, Grillner S (1993) Voltage clamp frequency domain analysis of NMDA activated neurons. J Exp Biol 175:59-87.

Moore LE, Buchanan JR, Murphey CR (1994) Anomalous increase in membrane impedance of neurons during NMDA activation. In: Computation in neurons and neural systems (Eeckman FH, ed), pp 9-14. Boston: Kluwer Academic.

Moore LE, Buchanan JT, Murphey CR (1995) Localization and interaction of NMDA and non-NMDA receptors in spinal neurons of the lamprey. Biophys J 68:96-103.

Müller W, Lux HD (1993) Analysis of voltage-dependent membrane currents in spatially extended neurons from point-clamp data. J Neurophysiol 69:241-247.

Murphey CR, Moore LE, Buchanan JT (1995) Quantitative analysis of electrotonic structure and membrane properties of NMDA activated lamprey spinal neurons. Neural Comput 7:486-506.

Murphey CR, Tabak J, Buchanan JT, Moore LE (1996) Estimation of membrane properties from step current measurements of Xenopus neurons. In: Computational neuroscience (Bower JM,ed) pp 107-112. New York: Academic.

O'Donovan MJ, Chub N (1997) Population behavior and selforganization in the genesis of spontaneous rhythmic activity by developing spinal networks. Semin Cell Dev Biol 8:21-28.

O'Donovan M, Sernagor E, Sholomenko G, Ho S, Antal M, Yee W (1992) Development of spinal motor networks in the chick embryo. J Exp Zool 261:261-273.

Rall W (1960) Membrane potential transients and membrane time constants of motoneurons. Exp Neurol 2:503-532.

Rall W, Segev I (1985) Space-clamp problems when voltage clamping branched neurons with intracellular electrodes. In: Voltage and patch clamping with microelectrodes (Smith TG, Lecar H, Redman SJ, Gage P, eds), pp 191-216. Bethesda, MD: American Physiological Society.

Rapp M, Segev I, Yarom Y (1994) Physiology, morphology and detailed passive models of guinea-pig cerebellar Purkinje cells. J Physiol (Lond) 474.1:101-118.

Roberts A, Tunstall MJ, Wolf E (1995) Properties of networks control- ling locomotion and significance of voltage dependency of NMDA channels: simulation study of rhythm generation sustained by positive feedback. J Neurophysiol 73:485-495.

Scrymgeour-Wedderburn JF, Reith CA, Sillar KT (1997) Voltage oscillations in Xenopus spinal cord neurons: developmental onset and dependence on co-activation of NMDA and 5HT receptors. Eur J Neurosci 9:1473-1482.

Segev I, Rinzel J, Shepherd GM (1995) The theoretical foundation of dendritic function: selected papers of Wilfred Rall. Cambridge, MA: MIT.

Sernagor E, O’Donovan MJ (1991) Whole-cell patch clamp recordings from rhythmically active motoneurons in the isolated spinal cord of the chick embryo. Neurosci Lett 128:211-216.

Sernagor E, Chub N, Ritter A, O’Donovan MJ (1995) Pharmacological characterization of the rhythmic synaptic drive onto lumbosacral motoneurons in the chick embryo spinal cord. J Neurosci 15:7452-7474.

Seutin V, Johnson SW, North RA (1994) Effect of dopamine and baclofen on $N$-methyl-D-aspartate-induced burst firing in rat ventral tegmental neurons. Neuroscience 58:201-206.

Spruston N, Johnston D (1992) Perforated patch-clamp analysis of the passive membrane properties of three classes of hippocampal neurons. J Neurophysiol 67:508-529.

Spruston N, Jaffe DB, Williams SH, Johnston D (1993) Voltage and space-clamp errors associated with the measurement of electrotonically remote synaptic events. J Neurophysiol 70:781-802.

Spruston N, Jaffe DB, Johnston D (1994) Dendritic attenuation of synaptic potentials and currents: the role of passive membrane properties. Trends Neurosci 17:161-166.

Stuart G, Sakmann B (1995) Amplification of EPSPs by axosomatic sodium channels in neocortical pyramidal neurons. Neuron 15:1065-1076.

Stuart G, Spruston N (1998) Determinants of voltage attenuation in neocortical pyramidal neuron dendrites. J. Neurosci 18:3501-3510.

Surmeier DJ, Wilson CJ, Eberwine J (1994) Patch-clamp techniques for studying potassium currents in mammalian brain neurons. Methods Neurosci 19:39-67.

Traub R, Miles R (1991) Neuronal networks of the hippocampus. New York: Cambridge UP.

Wallén P, Ekeberg C, Lansner A, Brodin L, Traven H, Grillner SA (1992) Computer-based model for realistic simulations of neural networks. II. The segmental network generating locomotor rhythmicity in the lamprey. J Neurophysiol 68:1939-1950.

White JA, Sekar NS, Kay AR (1995) Errors in persistent inward currents generated by space-clamp errors: a modeling study. J Neurophysiol 73:2369-2377.

Wilson CJ, Park MR (1989) Capacitance compensation and bridge balance adjustment in intracellular recording from dendritic neurons. J Neurosci Methods 27:51-75.

Wolfram S (1996) The Mathematica book. New York: Cambridge UP. Wright WN, Bardakjian BL, Valiante TA, Periz-Velazquez JL, Carlen P (1996) White noise approach for estimating the passive electrical properties of neurons. J Neurophysiol 76:3442-3450. 